\title{
Addition of erlotinib to fluoropyrimidine-oxaliplatin-based chemotherapy with or without bevacizumab: Two sequential phase I trials
}

\author{
CHIARA CARLOMAGNO*, GENNARO DANIELE*, ROBERTO BIANCO, ROBERTA MARCIANO, \\ VINCENZO DAMIANO, ELIDE MATANO, LUCIA NAPPI, STEFANO PEPE, \\ SABINO DE PLACIDO and GIAMPAOLO TORTORA
}

Department of Molecular and Clinical Endocrinology and Oncology, University of Naples Federico II, 80131 Naples, Italy

Received October 11, 2010; Accepted February 21, 2011

DOI: $10.3892 / \mathrm{etm} .2011 .218$

\begin{abstract}
The combination of EGFR inhibitors and antiangiogenic drugs has a strong pre-clinical rationale, yet its use has produced controversial clinical results. We conducted two sequential phase I trials to evaluate the feasibility and the recommended dose of erlotinib when combined with fluoropyrimidine-oxaliplatin-based chemotherapy with or without bevacizumab. A total of 21 metastatic colorectal cancer (mCRC) patients were treated in two sequential phase I trials. In the first trial, 12 patients were treated with escalating doses of erlotinib plus FOLFOX. In the second, 9 patients were treated with escalating doses of erlotinib combined with oxaliplatin, capecitabine and bevacizumab. No MTD was reached in either of the trials. The only dose-limiting toxicities observed were neutropenia and diarrhea. No unexpected toxicities were noted. Hematological toxicity was the most frequently noted adverse event with infusional 5FU therapy, while gastrointestinal toxicity was the most common adverse event. In the second trial most patients withdrew from treatment due to toxicity, and less than half completed the therapeutic program as per protocol, mostly due to toxicity. In conclusion, the present study confirms the disappointing results of the double combination of EGFR inhibitors and anti-angiogenic drugs in $\mathrm{mCRC}$ patients.
\end{abstract}

\section{Introduction}

Colorectal cancer (CRC) is the fourth most common malignancy worldwide (1). Nineteen percent of CRC patients are

Correspondence to: Dr Giampaolo Tortora, Department of Molecular and Clinical Endocrinology and Oncology, University of Naples Federico II, Via S. Pansini 5, 80131 Naples, Italy

E-mail: gtortora@unina.it

*Contributed equally

Key words: phase I trial, colorectal cancer, bevacizumab, erlotinib, capecitabine, FOLFOX diagnosed with metastatic disease, and $50 \%$ of those with local disease ultimately develop metastases; the 5-year survival rates are $90.8 \%$ for localized, $69.5 \%$ for regional and $11.3 \%$ for metastatic disease (2). However, due to the proper combination of active drugs, the survival of metastatic colorectal cancer (mCRC) patients has been increased to over 25 months.

The combination of oxaliplatin and capecitabine (XELOX) is considered a standard regimen for patients with $\mathrm{mCRC}$ based on the results of several studies. Recently, a randomized phase III trial designed to demonstrate the non-inferiority of XELOX vs. FOLFOX met its primary endpoint showing no difference between the two treatments with regard to response rate (RR), progression-free survival (PFS) and overall survival (OS) (3). Moreover, a meta-analysis of six phase II and III clinical trials, comparing 5FU- and capecitabine-based regimens, showed no difference in terms of PFS and OS between the two types of regimens, and a slight advantage in terms of RR for the 5FU-based therapy (4).

Angiogenesis is crucial to tumor growth and development of distant metastases (5); VEGF is a key player and a potential target for therapy (6). Since 2005, bevacizumab, a humanized monoclonal antibody directed against VEGF-A, has been integrated in the first-line therapy of $\mathrm{MCRC}$ patients based on the results of a phase III randomized trial by Hurwitz et al (7). The addition of bevacizumab to 5FU-irinotecan (IFL) therapy produced a significant increase in median OS when compared to IFL alone (20.3 vs. 15.6 months). When bevacizumab was added to first-line FOLFOX or XELOX therapy, a significant increase in PFS (9.4 vs. 8.0 months), median OS (21.3 vs. 19.9 months) and RR (47 vs. 49\%) was noted when compared to the chemotherapy alone (8). However, the shorter duration of therapy and the smaller number of patients receiving bevacizumab until disease progression in the latter study were claimed to be the main reasons for the lower strength of these results as compared to those found by Hurwitz et al.

The EGFR pathway plays a pivotal role in the growth and progression of the majority of human epithelial cancers, and its overexpression is associated with a poor prognosis (9). Monoclonal antibodies targeting EGFR have been approved to treat mCRC patients with a wild-type KRAS tumor, either in 


\section{M017974 trial}

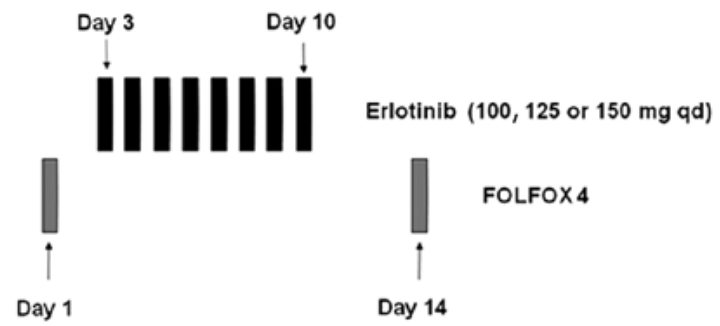

\section{ML18511 trial}

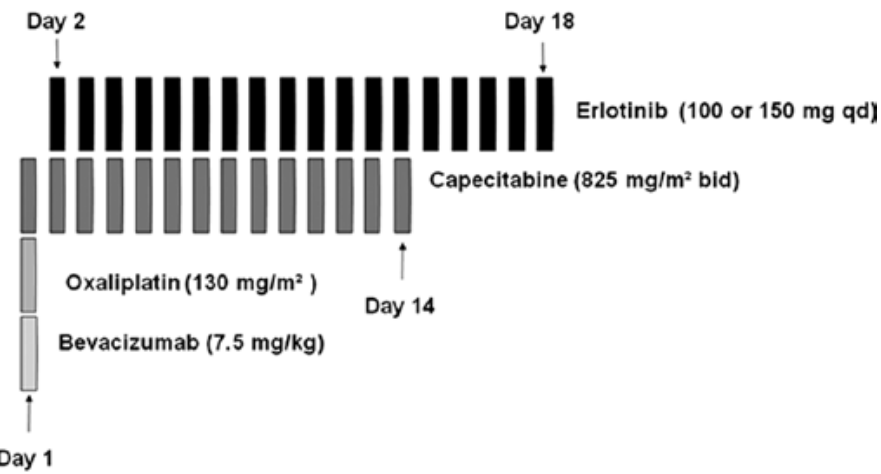

Figure 1. MO17974 trial: erlotinib, at escalating doses, administered orally from day 3 to day 10, was combined with FOLFOX4 administered every 14 days. ML18511 trial: erlotinib, at escalating doses, was administered on days 2-18 of each 21-day cycle combined with XELOX plus bevacizumab. qd, once a day; bid, twice a day.

combination with chemotherapy (10-12) or as a monotherapy $(13,14)$. Conversely, data are limited in regards to the use of erlotinib in combination with oxaliplatin-based therapy for either pre-treated (15) or chemo-naïve (16) mCRC patients. One study in which erlotinib was administered continuously at the dose of $150 \mathrm{mg}$ in combination with capecitabine and oxaliplatin to previously treated patients reported beneficial activity, but raised important safety issues. In fact, the first 13 patients enrolled in this study presented G3 diarrhea, leading to the reduction of the capecitabine dose from 2,000 to $1,500 \mathrm{mg} / \mathrm{m}^{2}(15)$. A second phase Ib trial identified the maximum tolerated dose (MTD) of erlotinib as $100 \mathrm{mg} /$ day administered continuously when combined with XELOX. In this trial, G3 diarrhea was the most commonly noted adverse event and the dose-limiting toxicity (DLT) (16).

Several pre-clinical studies have suggested that EGFR and VEGF are functionally linked (17). VEGF appears to be a preferred escape pathway to overcome the inhibitory effect of anti-EGFR agents in colon cancer cells with acquired resistance to anti-EGFR agents $(15,16)$. Consequently, the resistant cancer cells express 5- to 10-fold more VEGF-A and VEGFR-1 than the parental cells (18).

Pre-clinical studies suggest that erlotinib may have potential cooperative activity with fluoropyrimidines, oxaliplatin and bevacizumab. However, few clinical data are available in regards to the possibility of combining these agents.

Based on the above findings, we prospectively evaluated in two trials, MO17974 and ML18511 (EudraCT 2005005548-21), the feasibility of the addition of erlotinib to therapy with fluoropyrimidines and oxaliplatin, with or without bevacizumab.

\section{Patients and methods}

Patient eligibility. Patients with histologically confirmed $\mathrm{mCRC}$, previously untreated for metastatic disease, $\geq 18$ years of age, an ECOG performance status (PS) 0 to 1, with a life expectancy of $\geq 12$ weeks and at least one measurable lesion according to RECIST criteria (19) were eligible. All patients had normal organ function defined as: neutrophils $\geq 1,500$ and platelets $\geq 100,000$; total bilirubin $\leq 1.5 \times$ UNL, ASAT and ALAT $\leq 2.5 \times$ UNL ( $\leq 5$ in case of liver metastases), ALP $\leq 2.5$ $x$ UNL ( $<5$ in case of liver metastases; $\leq 10$ in case of bone metastases); creatinine $\leq 1.5 \times$ UNL or creatinine clearance $>50 \mathrm{ml} / \mathrm{min}$; and urine dip-stick proteinuria $<2+$.

Main exclusion criteria included: radiotherapy administered within 4 weeks before the start of the study, the presence of brain metastases, prior adjuvant therapy containing oxaliplatin, history of inflammatory bowel disease and or acute occlusion/sub-occlusion, presence of non-healing ulcers or coagulopathies, uncontrolled hypertension or clinically active cardiovascular disease, current and ongoing treatment with anticoagulant drugs, receiving other investigational drugs within 30 days before the study entry, or subjected to major surgical procedures within 28 days before the study entry.

Prior informed consent was obtained from all patients before enrollment in the study. The trials were conducted in accordance with the Declaration of Helsinki and the latest version of the ICH Harmonized Tripartite Guideline: Guideline for Good Clinical Practice and local laws and regulations. The protocols were approved by the Ethics Committee of the University Federico II of Naples. Both trials were designed, implemented and analyzed independently by the investigators.

Treatment plan. A classical $3+3$ dose escalation design was used for both trials.

The first trial (MO17974) evaluated the combination of fixed doses of FOLFOX4 with escalating doses of erlotinib $(100,125$ and $150 \mathrm{mg} /$ day) administered orally from day 3 to day 10 of each 14-day cycle (Fig. 1).

In the second trial (ML18511), escalating doses of erlotinib (100 and $150 \mathrm{mg} /$ day, on days 2-18) were combined with fixed doses of capecitabine $\left(1,650 \mathrm{mg} / \mathrm{m}^{2}\right.$, on days $\left.1-14\right)$ and oxaliplatin $\left(130 \mathrm{mg} / \mathrm{m}^{2}\right.$, day 1) (XELOX) plus bevacizumab $(7.5 \mathrm{mg} /$ $\mathrm{kg}$, day 1). Treatment was repeated every 21 days (Fig. 1). The initial dose of erlotinib in both trials was lower than the MTD reported in the literature to minimize potential side effects.

Patients received up to 6 months of combined treatment (12 cycles in the FOLFOX trial or 9 cycles in the XELOX trial). Thereafter, they continued treatment with erlotinib or with erlotinib plus bevacizumab until disease progression or intolerable toxicity. 
Dose escalation, dose-limiting toxicities and definitions of the maximum tolerated dose. Three patients were sequentially treated at each dose level (DL). DL was expanded to six when a DLT was observed during the first cycle. Dose escalation was continued when $<33 \%$ of patients experienced a DLT. In the event that $\geq 2 / 3$ or $\geq 2 / 6$ of patients experienced a DLT, the MTD was considered to be exceeded.

DLT was reached when any of the following events occurred in the first cycle of treatment: i) febrile neutropenia for $>3$ days, ii) G4 neutropenia lasting $>7$ days, iii) G4 thrombocytopenia, or iv) any drug-related non-hematological toxicity $\geq \mathrm{G} 3$ (apart from alopecia). The MTD was defined as the dose below that which caused DLT in $>33 \%$ of patients treated at a DL.

Patient monitoring and response assessment. Pre-treatment assessment within 2 weeks of the start of treatment included receipt of informed consent, evaluation of demographic information, procurement of a full medical history, general physical examination, ECG, laboratory tests (including blood chemistry and urine analysis) and total body CT-scan. Chest X-ray was required when a CT-scan of the chest was unavailable.

A clinical examination was performed at each cycle of treatment. Laboratory analyses were performed weekly. The severity of all the adverse events and abnormal laboratory results were graded according to the CTCAE v3.0.

Reassessment imaging was performed every 4 cycles for FOLFOX and 3 cycles for XELOX, or as required when progressive disease (PD) was suspect. Responses were evaluated according to the RECIST guidelines (19).

Materials. Roche kindly supplied erlotinib for both the trials (MO17974 and ML18511) and bevacizumab for the ML18511 trial.

\section{Results}

\section{Patient characteristics and treatment}

MO17974 trial. From June 2004 to July 2005, 12 patients were enrolled in the trial (Table I). Seventy-five percent of the patients had $\geq 2$ sites of metastatic disease, most of them involving the liver. Overall, 100 cycles of chemotherapy were administered, with a median of 9.5 cycles/patient (range 2-12). Three of the 12 enrolled patients discontinued treatment due to disease progression (after cycle 6, 8 and 9); 4 patients discontinued chemotherapy due to toxicity (1 unacceptable toxicity after cycle $1 ; 1$ after cycle 3 due to G2 hyperbilirubinemia; 1 after cycle 7 for prolonged G3 thrombocytopenia and 1 after cycle 11 due to G3 peripheral neuropathy and mucositis). Five patients, after the completion of chemotherapy, continued with erlotinib treatment alone for a median of 3.7 months (range 1-8.8) until disease progression.

ML18511 trial. From June 2006 to June 2007, 9 patients were enrolled in the trial (Table I). All patients completed at least 1 cycle of therapy. A total number of 51 cycles of therapy was delivered with a median of 3 per patient (range 1-19). One patient at DL 1 and one at DL 2 received further cycles (3 and 10 cycles, respectively) of erlotinib and bevacizumab after the completion of the first 9 cycles of therapy. Three patients at DL 1 withdrew from treatment due to PD after 12, 3 and
Table I. Patient characteristics.

\begin{tabular}{lcc}
\hline & $\begin{array}{c}\text { MO17974 trial } \\
(\mathrm{n}=12)\end{array}$ & $\begin{array}{c}\text { ML18511 trial } \\
(\mathrm{n}=9)\end{array}$ \\
\hline Age (years) & & \\
$18-64$ & 8 & 5 \\
$\geq 65$ & & 4 \\
Gender & 8 & 6 \\
Male & 4 & 3 \\
Female & & \\
Baseline PS (WHO) & 11 & 6 \\
0 & 1 & 3 \\
1 & - & - \\
2 & & 8 \\
Site of metastasis & 10 & 4 \\
Liver & 1 & 7 \\
Lung & 7 & 1 \\
Node & 2 & \\
Other & (peritoneum) & (serratus muscle) \\
& &
\end{tabular}

PS, ECOG performance status.

5 cycles of therapy, respectively. Five patients withdrew due to toxicity: 3 at DL 1 (1 patient due to rectal bleeding at cycle 5, and 2 patients due to G4 diarrhea at cycle 2 and 3, respectively) and 2 at DL 2 (due to G4 diarrhea experienced at cycle 1 and 2). One patient withdrew on a voluntary basis after 19 cycles, although she experienced only mild toxicity, consisting of G2 rectal bleeding.

\section{Toxicity}

MO17974 trial. At DL 1 (erlotinib $100 \mathrm{mg}$ ) and 2 (erlotinib $125 \mathrm{mg}$ ), no unacceptable toxicity was noted during the first cycle of treatment. At DL 3 (erlotinib $150 \mathrm{mg}$ ), 1/6 of the enrolled patients experienced unacceptable toxicity at the first cycle of treatment, consisting of G3 diarrhea and G3 neutropenia. Thus, the MTD was not reached.

The most severe side effects experienced by the 12 enrolled patients throughout treatment are listed in Tables II and III. Non-hematological toxicity was mild. In addition to the episodes of unacceptable toxicity reported above (G3 diarrhea), only 1 patient experienced $\geq \mathrm{G} 3$ gastrointestinal toxicity (mucositis); $\geq \mathrm{G} 2$ peripheral neuropathy occurred in 2 patients and was related to the cumulative administered dose of oxaliplatin, as it appeared after the eighth cycle of chemotherapy. As expected with the FOLFOX regimen, hematological toxicity was frequent: $50 \%$ of patients experienced G3-4 neutropenia and 2 patients presented with G3 thrombocytopenia.

G1-2 skin reactions as acneiform dermatitis, very likely related to EGFR inhibition by erlotinib, were observed in 50\% of the treated patients, but in no cases were delay or discontinuation of erlotinib administration required.

ML18511 trial. No DLT was observed at DL 1, while at DL 2, 1 patient experienced a DLT consisting of G4 diarrhea. Most common toxicities occurring during the first cycle consisted of diarrhea, nausea and vomiting, skin rash, 


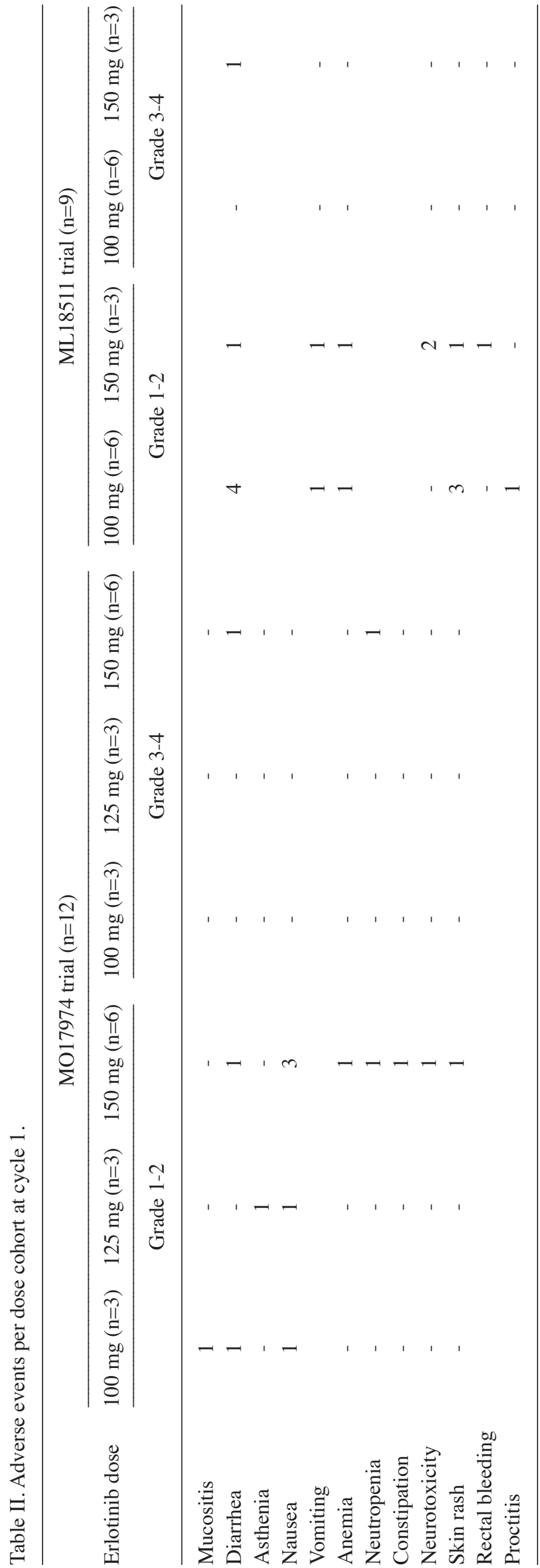

paresthesia and rectal bleeding (Table II). Their entity was moderate and did not require a treatment delay. Table III summarizes the toxicity observed at cycles other than 1 . The most common adverse event was diarrhea. In 2 cases, 1 at DL 1 and 1 at DL 2, diarrhea was severe and required medical therapy. The incidence of nausea and vomiting was lower than expected and was severe in 1 patient at DL 1 . One patient at DL 1 experienced hypersensitivity during bevacizumab administration, consisting in a spasm of the larynx and requiring medical treatment. Two patients, 1 at DL 1 and 1 at DL 2, experienced rectal bleeding, which was complicated by G3 anemia in the patient at DL 1 . Only 1 patient at DL 1 and 1 at DL 2 experienced G2 neutropenia, after cycle 6 and 3 of therapy, respectively. Three patients at DL 1 experienced a mild increase in liver enzymes.

\section{Tumor response}

MO17479 trial. All patients were assessable for tumor response: at DL 1,2 patients obtained a partial response (PR) and 1 stable disease (SD); at DL 2, 1 patient experienced a PR and 2 SD. Five SD and 1 PD cases were noted at DL 3 (Table IV). Ten patients received further chemotherapy after disease progression (mostly an irinotecan-containing regimen with or without cetuximab); 5 patients received $\geq 2$ lines of additional chemotherapy.

ML18511 trial. Six patients at DL 1 and 2 patients at DL 2 were evaluable for tumor response. Three patients at DL 1 and 1 patient at DL 2 experienced a PR; 2 SD cases were noted at DL 1 and 2, respectively. Two patients at DL 1 experienced PD as the best response (Table IV). All patients received at least a second-line chemotherapy regimen. One patient at DL 2 underwent liver metastasectomy after second-line chemotherapy. Seven patients received $\geq 2$ lines of therapy after the trial.

\section{Discussion}

Oxaliplatin-based therapy is at present considered a standard first-line treatment for mCRC patients. Since the addition of cetuximab to FOLFOX therapy has demonstrated substantial efficacy in the treatment of mCRC patients $(12,20)$ and erlotinib has showed beneficial activity as a monotherapy in first-line mCRC therapy (21), a combination of erlotinib and FOLFOX in a first-line setting may represent an appealing treatment option.

In both the present trials, MTD was not reached. Our first trial, MO174974, found that FOLFOX-erlotinib is a feasible treatment for first-line $\mathrm{mCRC}$, since the combination demonstrated a good safety profile, with no synergism of toxicity between the drugs and an acceptable median number of cycles delivered (9 cycles). The results are consistent with the encouraging results of two recently published phase I studies, which demonstrated a satisfactory toxicity profile and beneficial activity for erlotinib combined with oxaliplatin and fluoropyrimidines $(16,22)$.

In a study by Hanauske et al, (22) the MTD of erlotinib was found to be $150 \mathrm{mg}$ when administered continuously with FOLFOX in 32 patients with advanced solid tumors (including 23 with $\mathrm{mCRC}$ ). The most common adverse events included diarrhea, nausea, stomatitis and rash. Among the mCRC patients, 1 obtained a complete response (CR) and 7 a PR. In another trial (16), the MTD of erlotinib was determined to be $100 \mathrm{mg}$ when administered continuously with 


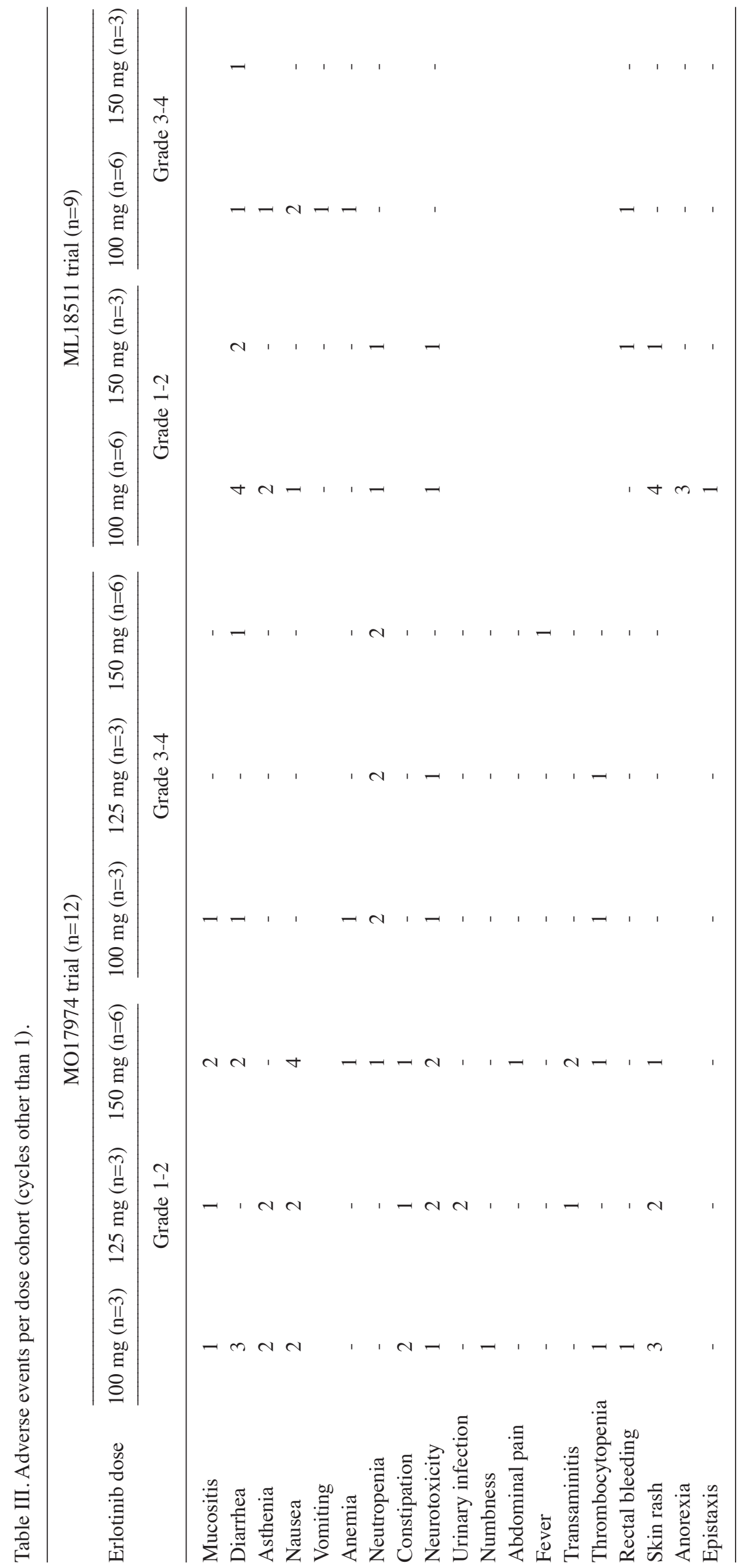


Table IV. Clinical outcome.

\begin{tabular}{lcccc}
\hline & \multicolumn{3}{c}{ MO17974 trial } & ML18511 trial \\
\cline { 2 - 5 } & & & Erlotinib dose (mg) & \\
\cline { 2 - 5 } Response & 100 & 125 & 150 & 100 \\
\hline PR & 2 & 1 & - & 3 \\
SD & 1 & 2 & 5 & 1 \\
PD & - & - & 1 & 2 \\
\hline
\end{tabular}

${ }^{a}$ One patient in this cohort was not evaluable for response. PR, partial response; SD, stable disease; PD, progressive disease.

XELOX. The most common adverse events were diarrhea, rash, nausea and neurotoxicity, with an expected dose-dependent trend. The rate of rash and diarrhea prevented any further escalation to higher doses of erlotinib and capecitabine.

In our second trial, ML18511, the most common toxicity was diarrhea, thus confirming the finding of previous studies $(23,24)$. Although the treatment regimen resulted in only 1 DLT at the first cycle, in subsequent cycles almost all patients experienced a moderate/severe toxicity that variably hampered daily living activities and forced patient withdrawal from treatment. The median number of administered cycles of therapy was very low ( 3 cycles) considering the first-line setting.

Previous studies have also explored this combination as a first-line therapy of mCRC patients. In a phase II trial by Spigel et al (23) the combination of erlotinib (150 mg) administered continuously with FOLFOX-4 and bevacizumab every 14 days caused G3 diarrhea in 31\% of patients and G3 nausea in 14\% of patients. Moreover, there were four deaths possibly related to treatment. For these reasons, the study was discontinued.

Meyerhardt et al published the results of a phase II trial combining mFOLFOX-6 plus bevacizumab with continuous administration of erlotinib (150 mg) (24). The primary endpoint of the trial, PFS, was not reached, partly due to the toxicity observed. In fact, 7 patients discontinued the trial due to toxicity before the first restaging: 4 of them voluntarily withdrew due to uncomfortable moderate toxicity, and 3 due to severe toxicity. In total, $77 \%$ of the patients exited the study due to toxic effects or withdrawal of informed consent. The toxicity with the most impact was diarrhea (83\% of patients). Fifty-four percent of the patients required at least one dose reduction of erlotinib, and 4 patients requiring two dose reductions (final erlotinib dose, $50 \mathrm{mg}$ daily).

Although treatment activity was beyond the scope of our trial, all patients were followed up for evaluation of tumor response. Four patients (50\%) obtained a PR, 3 at the first DL and 1 at the second DL, and only 2 patients had PD. These data are comparable to those of the above-mentioned two phase II studies, in which PR was obtained in $43 \%$ (23) and $34 \%$ of the patients (24), respectively.

Current evidence shows that severe or uncomfortable non-acute toxicity is the main concern regarding the combination of bevacizumab and erlotinib with fluoropyrimidine and oxaliplatin chemotherapy. Even though these early data show promising activity for this combination, recently published phase III trials $(25,26)$ have raised the question of whether the double blockade of EGFR and VEGF by two monoclonal antibodies is an effective treatment for mCRC patients. In fact, in the CAIRO-2 study, the combination of cetuximab, bevacizumab and CAPOX resulted in a significant decrease in PFS, the primary endpoint of the trial (9.7 vs. 10.4 months, $\mathrm{p}=0.001$ ), when compared to that of CAPOX plus bevacizumab (26). In the PACCE study, the addition of panitumumab to bevacizumab plus oxaliplatin or irinotecan-based chemotherapy resulted in a worse PFS vs. the same therapy without panitumumab (25). A possible explanation for the disappointing results achieved in these studies, as compared to pre-clinical findings, is the decreased amount of therapy administered to the patients receiving the triple-combination therapy, due to toxicity.

It has recently been pointed out that only KRAS wild-type patients are able to benefit from anti-EGFR therapies. However, the KRAS or EGFR mutational status of our patients was unknown. Thus, we could not draw any conclusions regarding the degree of activity in this subgroup of patients who are the best candidates for anti-EGFR therapy.

Therefore, based on the safety profile observed in our and previous studies investigating the combination of erlotinib with fluoropyrimidine-based chemotherapy, and on the concerns raised by studies which combined anti-EGFR antibodies with bevacizumab plus chemotherapy, we conclude that combining an anti-EGFR TKI, such as erlotinib, with chemotherapy and bevacizumab may be unsuitable for the treatment of mCRC patients.

\section{Acknowledgements}

This study was supported in part by a grant from the Associazione Italiana Ricerca sul Cancro (AIRC) and Regione Campania.

\section{References}

1. Jemal A, Siegel R, Ward E, Hao Y, Xu J and Thun MJ: Cancer Statistics, 2009. CA Cancer J Clin 59: 225-249, 2009.

2. Horner MJ Ries LAG, Krapcho M, Neyman N, Aminou R, Howlader N, Altekruse SF, Feuer EJ, Huang L, Mariotto A, Miller BA, Lewis DR, Eisner MP, Stinchcomb DG and Edwards BK (eds): SEER Cancer Statistics Review, 1975-2006, National Cancer Institute. Bethesda, MD, http://seer.cancer.gov/ csr/1975_2006/, based on November 2008 SEER data submission, posted to the SEER web site, 2009. 
3. Cassidy J, Clarke S, Diaz-Rubio E, et al: Randomized phase III study of capecitabine plus oxaliplatin compared with fluorouracil/folinic acid plus oxaliplatin as first-line therapy for metastatic colorectal cancer. J Clin Oncol 26: 2006-2012, 2008.

4. Arkenau HT, Arnold D, Cassidy J, et al: Efficacy of oxaliplatin plus capecitabine or infusional fluorouracil/leucovorin in patients with metastatic colorectal cancer: a pooled analysis of randomized trials. J Clin Oncol 26: 5910-5917, 2008.

5. Hanahan D and Folkman J: Patterns and emerging mechanisms of the angiogenic switch during tumorigenesis. Cell 86: 353-364 1996.

6. Ferrara N, Gerber HP and LeCouter J: The biology of VEGF and its receptors. Nat Med 9: 669-676, 2003.

7. Hurwitz H, Fehrenbacher L, Novotny W, et al: Bevacizumab plus irinotecan, fluorouracil, and leucovorin for metastatic colorecta cancer. N Engl J Med 350: 2335-2342, 2004.

8. Saltz LB, Clarke S, Diaz-Rubio E, et al: Bevacizumab in combination with oxaliplatin-based chemotherapy as first-line therapy in metastatic colorectal cancer: a randomized phase III study. J Clin Oncol 26: 2013-2019, 2008.

9. Ciardiello $\mathrm{F}$ and Tortora G: EGFR antagonists in cancer treatment. N Engl J Med 358: 1160-1174, 2008.

10. Van Cutsem E, Kohne CH, Hitre E, et al: Cetuximab and chemotherapy as initial treatment for metastatic colorectal cancer. $\mathrm{N}$ Engl J Med 360: 1408-1417, 2009.

11. Cunningham D, Humblet Y, Siena S, et al: Cetuximab monotherapy and cetuximab plus irinotecan in irinotecan-refractory metastatic colorectal cancer. N Engl J Med 351: 337-345, 2004

12. Bokemeyer C, Bondarenko I, Makhson A, et al: Fluorouracil, leucovorin, and oxaliplatin with and without cetuximab in the first-line treatment of metastatic colorectal cancer. J Clin Oncol 27: 663-671, 2009.

13. Jonker DJ, O'Callaghan CJ, Karapetis CS, et al: Cetuximab for the treatment of colorectal cancer. N Engl J Med 357: 2040-2048, 2007.

14. Van Cutsem E, Peeters M, Siena S, et al: Open-label phase III tria of panitumumab plus best supportive care compared with best supportive care alone in patients with chemotherapy-refractory metastatic colorectal cancer. J Clin Oncol 25: 1658-1664, 2007.

15. Meyerhardt JA, Zhu AX, Enzinger PC, et al: Phase II study of capecitabine, oxaliplatin, and erlotinib in previously treated patients with metastastic colorectal cancer. J Clin Oncol 24: 1892-1897, 2006
16. Van Cutsem E, Verslype C, Beale P, et al: A phase Ib dose-escalation study of erlotinib, capecitabine and oxaliplatin in metastatic colorectal cancer patients. Ann Oncol 19: 332-339, 2008.

17. Bianco R, Rosa R, Damiano V, et al: Vascular endothelial growth factor receptor- 1 contributes to resistance to anti-epidermal growth factor receptor drugs in human cancer cells. Clin Cancer Res 14: 5069-5080, 2008.

18. Ciardiello F, Bianco R, Caputo R, et al: Antitumor activity of ZD6474, a vascular endothelial growth factor receptor tyrosine kinase inhibitor, in human cancer cells with acquired resistance to antiepidermal growth factor receptor therapy. Clin Cancer Res 10: 784-793, 2004

19. Therasse P, Arbuck SG, Eisenhauer EA, et al: New guidelines to evaluate the response to treatment in solid tumors. European Organization for Research and Treatment of Cancer, National Cancer Institute of the United States, National Cancer Institute of Canada. J Natl Cancer Inst 92: 205-216, 2000.

20. Tabernero J, van Cutsem E, Diaz-Rubio E, et al: Phase II trial of cetuximab in combination with fluorouracil, leucovorin, and oxaliplatin in the first-line treatment of metastatic colorectal cancer. J Clin Oncol 25: 5225-5232, 2007.

21. Townsley CA, Major P, Siu LL, et al: Phase II study of erlotinib (OSI-774) in patients with metastatic colorectal cancer. Br J Cancer 94: 1136-1143, 2006.

22. Hanauske AR, Cassidy J, Sastre J, et al: Phase $1 \mathrm{~b}$ dose escalation study of erlotinib in combination with infusional 5-fluorouracil, leucovorin, and oxaliplatin in patients with advanced solid tumors. Clin Cancer Res 13: 523-531, 2007.

23. Spigel DR, Hainsworth JD, Burris HA, et al: Phase II of FOLFOX4, bevacizumab and erlotinib as first-line therapy in patients with advanced colorectal cancer. In: ASCO Gastrointestinal Cancer Symposium: abs. 238, 2006

24. Meyerhardt JA, Stuart K, Fuchs CS, et al: Phase II study of FOLFOX, bevacizumab and erlotinib as first-line therapy for patients with metastatic colorectal cancer. Ann Oncol 18: 1185-1189, 2007

25. Hecht JR, Mitchell E, Chidiac T, et al: A randomized phase IIIB trial of chemotherapy, bevacizumab, and panitumumab compared with chemotherapy and bevacizumab alone for metastatic colorectal cancer. J Clin Oncol 27: 672-680, 2009.

26. Tol J, Koopman M, Cats A, et al: Chemotherapy, bevacizumab, and cetuximab in metastatic colorectal cancer. N Engl J Med 360: $563-572,2009$ 